\title{
Densification of ICRS in the Optical by use of Old Pulkovo Observation Sets
}

\author{
Irina I. Kumkova \\ Institute of Applied Astronomy RAS, 10, Kutuzov emb, St. Petersburg \\ 191187 Russia \\ Vadim V. Bobylev, Nina M. Bronnikova \\ Main Astronomical Observatory, Pulkovo, St.Petersburg, 196240, Russia
}

\begin{abstract}
Modern tasks of high precision astrometry demand optical coordinate systems including more faint stars than are available now in current conventional systems (Hipparcos). For this purpose it is suggested to use old photographic observations accumulated in the Pulkovo Observatory. Extensive observational data have been obtained at Pulkovo Observatory during the last century in the framework of several programs, e.g. Pulkovo Galaxy plan, etc. Observations have been made with the Normal Astrograph from 1894 to the present. The data are investigated with the aim of extending the Hipparcos catalog to stars fainter than 11th magnitude. All available observations are taken into account. A description of the material considered is given. The distribution of the selected plates over the celestial sphere is shown as well. Coordinates of faint stars in the Hipparcos system are calculated for selected areas. The accuracy of computed star coordinates is analyzed. Results of the investigation are presented.
\end{abstract}

\section{Introduction}

At the first astrometric conference (Pulkovo, 1932) an idea was proposed to compile the Fundamental Catalog of Faint Stars, and to use galaxies to determine absolute star proper motions. Later (in 1937) Prof. A.N. Deutsch demonstrated the possibility of connecting stars to galaxy NGC 4262 to determine absolute star proper motions. A list of areas with galaxies was compiled for such a goal by P. G. Kulikovsky and G. N. Neuimin in 1940. It consisted of 143 areas with 271 galaxies. The list was checked in Pulkovo, Moscow and Tashkent observatories. Regular observations of areas with galaxies started in Pulkovo observatory in 1939. The observations stopped during the Second World War and the following period of restoration from 1941 to 1949 . Before the war, 123 plates with galaxies were obtained in Pulkovo and 94 plates in Tashkent from 1941 to 1949 . In 1950 the observational list was re-checked. Some areas were excluded and several were added. In Pulkovo the observations were carried out using the Pulkovo Normal Astrograph $\left(\mathrm{F}=3.5 \mathrm{~m}, \mathrm{D}=33 \mathrm{~cm}\right.$, plate $\left.2^{\circ} \times 2^{\circ}\right)$. As a result of all long-term work the catalog - PUL2 of 59646 star proper motions 
with respect to galaxies was completed for 149 areas of the northern hemisphere in 1999. The areas are distributed uniformly over declinations from $-5^{\circ}$ to $+85^{\circ}$. The first epoch of the photographic plates was obtained from 1937 to 1965 , and the period of the second one is from 1969 to 1986. The mean difference of epochs is equal to 24 years. Three pairs of photographic plates were obtained for every area. All plates were taken with 1-hour and 5-minutes exposures. Bright stars were measured only on plates taken with a diffraction grating. Relative star proper motions were calculated using faint (mag 15) reference stars. A sixconstant model has been used in the reduction of plates. 700 galaxies were used. Random errors of relative star proper motions were estimated for 18250 stars and are $5.5 \mathrm{mas} / \mathrm{yr}$ (milliarcsecond per year) for $\mu_{\alpha} \cos \delta$ and $5.9 \mathrm{mas} / \mathrm{yr}$ for $\mu_{\delta}$. Errors of fictitious proper motions of galaxies are 7.9 mas/yr (mean calibration error) for both coordinates. The mean random error of absolute bright star proper motions are equal to $9.3 \mathrm{mas} / \mathrm{yr}$ for $\mu_{\alpha} \cos \delta$ and $9.4 \mathrm{mas} / \mathrm{yr}$ for $m u_{\delta}$ derived from comparison of PUL2 and Hipparcos catalogs. Mean random errors of absolute faint star (41000 stars) proper motions in PUL2 are $7.9 \mathrm{mas} / \mathrm{yr}$ for both coordinates.

\section{Calculations}

The calculations were carried out for 5 areas selected from PUL2 for pilot work. Two areas are presented at Figs. 1-2. After reduction of star coordinates to the same epoch for both catalogs PUL2 and Hipparcos, an identification of common stars has been done. A six-constant model has been applied to the reduction of the plates. Hipparcos stars were used as references. The calculations were performed for 30 PUL2 stars. The resulting equatorial coordinates of PUL2 stars in different areas are presented in Tables 1-3.

\section{Conclusions}

It is possible to use PUL2 - the catalog of 59646 stars proper motions with respect to galaxies - to extend the Hipparcos catalog to faint stars in the selected areas without significant loss of accuracy. Moreover the results of such an extension will give more material for the analysis of Galaxy kinematics. 


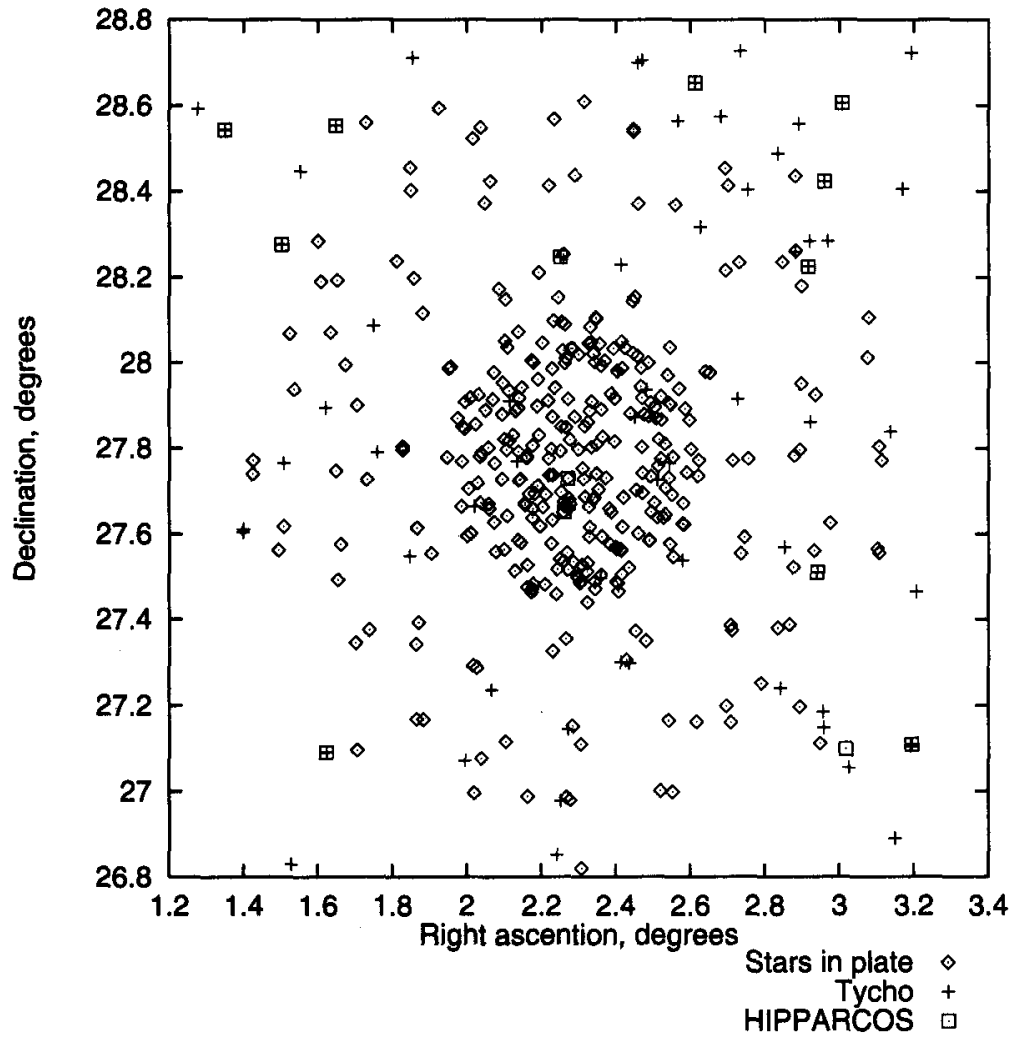

Figure 1. 


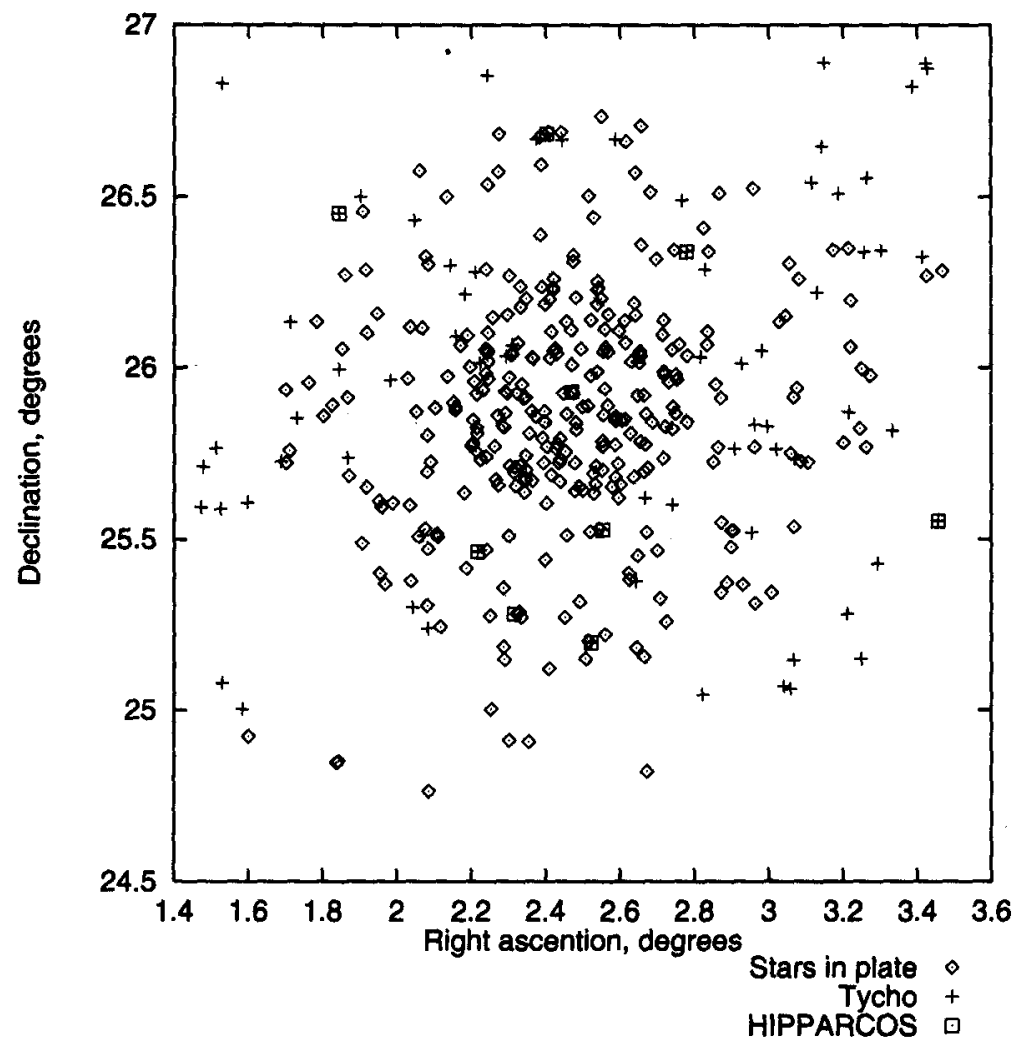

Figure 2. 
Table 1. $\alpha=00^{h} 09^{m} 04^{s} \cdot 920, \delta=27^{\circ} 43^{\prime} 41^{\prime \prime} .52$

$\begin{array}{rcccccccc}\text { PUL2 Number } & \text { hh } & \text { mm } & \text { ss } & \sigma_{\alpha}, \mathrm{ss} & \delta, \mathrm{deg} & \delta, \mathrm{mm} & \delta, \mathrm{ss} & \sigma_{\delta}, \mathrm{ss} \\ 432 & 00 & 06 & 24.184 & 0.0009 & 27 & 58 & 16.41 & 0.002 \\ 10 & 00 & 06 & 53.303 & 0.0007 & 28 & 10 & 3.60 & 0.003 \\ 324 & 00 & 07 & 26.962 & 0.0005 & 27 & 26 & 6.79 & 0.002 \\ 206 & 00 & 07 & 53.121 & 0.0004 & 27 & 48 & 5.87 & 0.001 \\ 351 & 00 & 08 & 02.829 & 0.0003 & 27 & 29 & 57.48 & 0.001 \\ 201 & 00 & 08 & 08.232 & 0.0003 & 27 & 45 & 28.62 & 0.002 \\ 284 & 00 & 08 & 16.132 & 0.0003 & 27 & 51 & 28.75 & 0.001 \\ 285 & 00 & 08 & 23.278 & 0.0002 & 27 & 53 & 46.68 & 0.001 \\ 218 & 00 & 08 & 30.447 & 0.0002 & 27 & 48 & 38.69 & 0.001 \\ 124 & 00 & 08 & 36.404 & 0.0001 & 27 & 41 & 48.56 & 0.001 \\ 52 & 00 & 08 & 41.152 & 0.0001 & 27 & 35 & 31.22 & 0.001 \\ 190 & 00 & 08 & 45.137 & 0.0001 & 27 & 46 & 48.37 & 0.004 \\ 150 & 00 & 08 & 51.872 & 0.0001 & 27 & 43 & 49.72 & 0.001 \\ 276 & 00 & 08 & 55.477 & 0.0001 & 27 & 50 & 17.93 & 0.001 \\ 464 & 00 & 09 & 00.583 & 0.0001 & 27 & 41 & 47.48 & 0.001 \\ 318 & 00 & 09 & 02.795 & 0.0004 & 27 & 20 & 19.68 & 0.002 \\ 272 & 00 & 09 & 07.026 & 0.0002 & 27 & 53 & 10.15 & 0.001 \\ 332 & 00 & 09 & 11.894 & 0.0005 & 27 & 24 & 10.80 & 0.002 \\ 40 & 00 & 09 & 16.353 & 0.0004 & 27 & 34 & 30.56 & 0.001 \\ 184 & 00 & 09 & 18.866 & 0.0003 & 27 & 45 & 53.64 & 0.001 \\ 158 & 00 & 09 & 22.015 & 0.0001 & 27 & 43 & 54.63 & 0.001 \\ 182 & 00 & 09 & 26.418 & 0.0001 & 27 & 46 & 40.26 & 0.001 \\ 80 & 00 & 09 & 34.616 & 0.0002 & 27 & 38 & 32.55 & 0.001 \\ 307 & 00 & 09 & 38.850 & 0.0002 & 27 & 53 & 40.16 & 0.001 \\ 452 & 00 & 09 & 46.723 & 0.0002 & 28 & 9 & 14.62 & 0.003 \\ 162 & 00 & 09 & 51.762 & 0.0003 & 27 & 43 & 56.43 & 0.001 \\ 242 & 00 & 09 & 57.437 & 0.0003 & 27 & 49 & 9.29 & 0.006 \\ 245 & 00 & 10 & 4.202 & 0.0003 & 27 & 49 & 37.21 & 0.007 \\ 247 & 00 & 10 & 9.749 & 0.0004 & 27 & 49 & 5.60 & 0.006 \\ 96 & 00 & 10 & 18.269 & 0.0004 & 27 & 41 & 44.02 & 0.002\end{array}$


Table 2. $\alpha=00^{h} 09^{m} 52^{s} .944, \delta=25^{\circ} 55^{\prime} 41^{\prime \prime} .52$

$\begin{array}{rcccccccc}\text { PUL2 Number } & \text { hh } & \text { mm } & \text { ss } & \sigma_{\alpha}, \text { ss } & \delta, \text { deg } & \delta, \text { mm } & \delta, \text { ss } & \sigma_{\delta}, \text { ss } \\ 357 & 00 & 08 & 29.212 & 0.0005 & 25 & 53 & 06.20 & 0.002 \\ 326 & 00 & 08 & 38.591 & 0.0004 & 25 & 56 & 17.49 & 0.001 \\ 369 & 00 & 09 & 00.556 & 0.0003 & 25 & 49 & 59.13 & 0.006 \\ 330 & 00 & 09 & 01.315 & 0.0003 & 25 & 55 & 38.60 & 0.001 \\ 172 & 00 & 09 & 12.315 & 0.0002 & 25 & 51 & 52.85 & 0.004 \\ 111 & 00 & 09 & 19.178 & 0.0002 & 25 & 54 & 28.49 & 0.001 \\ 10 & 00 & 09 & 19.611 & 0.0002 & 25 & 58 & 39.68 & 0.004 \\ 257 & 00 & 09 & 18.696 & 0.0002 & 26 & 01 & 14.31 & 0.006 \\ 104 & 00 & 09 & 30.728 & 0.0001 & 25 & 53 & 49.79 & 0.002 \\ 393 & 00 & 09 & 38.410 & 0.0001 & 25 & 47 & 55.01 & 0.008 \\ 161 & 00 & 09 & 36.899 & 0.0001 & 25 & 52 & 30.69 & 0.003 \\ 68 & 00 & 09 & 36.841 & 0.0001 & 25 & 55 & 49.83 & 0.001 \\ 196 & 00 & 09 & 43.863 & 0.0001 & 25 & 51 & 03.60 & 0.005 \\ 230 & 00 & 09 & 50.275 & 0.0001 & 25 & 49 & 00.91 & 0.007 \\ 215 & 00 & 09 & 51.716 & 0.0001 & 25 & 50 & 04.27 & 0.006 \\ 46 & 00 & 09 & 49.259 & 0.0001 & 25 & 55 & 54.82 & 0.001 \\ 190 & 00 & 09 & 57.861 & 0.0003 & 25 & 51 & 20.30 & 0.004 \\ 40 & 00 & 09 & 56.002 & 0.0002 & 25 & 56 & 05.89 & 0.001 \\ 156 & 00 & 10 & 04.384 & 0.0002 & 25 & 51 & 44.62 & 0.004 \\ 234 & 00 & 10 & 10.108 & 0.0001 & 25 & 48 & 04.04 & 0.008 \\ 152 & 00 & 10 & 09.017 & 0.0001 & 25 & 52 & 15.83 & 0.003 \\ 123 & 00 & 10 & 12.009 & 0.0001 & 25 & 53 & 20.43 & 0.002 \\ 419 & 00 & 10 & 25.756 & 0.0002 & 25 & 42 & 35.76 & 0.003 \\ 120 & 00 & 10 & 20.606 & 0.0002 & 25 & 52 & 30.20 & 0.003 \\ 52 & 00 & 10 & 20.255 & 0.0002 & 25 & 55 & 28.12 & 0.001 \\ 289 & 00 & 10 & 22.058 & 0.0002 & 25 & 58 & 31.39 & 0.003 \\ 80 & 00 & 10 & 31.907 & 0.0002 & 25 & 53 & 45.66 & 0.001 \\ 385 & 00 & 10 & 50.679 & 0.0003 & 25 & 45 & 53.08 & 0.005 \\ 288 & 00 & 10 & 46.375 & 0.0003 & 25 & 57 & 21.63 & 0.002 \\ 277 & 00 & 11 & 02.152 & 0.0004 & 26 & 00 & 04.59 & 0.005\end{array}$


Table 3. $\alpha=00^{h} 42^{m} 13^{s} 872, \delta=40^{\circ} 41^{\prime} 26^{\prime \prime} 52$

$\begin{array}{rcccccccc}\text { PUL2 Number } & \text { hh } & \mathrm{mm} & \mathrm{ss} & \sigma_{\alpha}, \mathrm{ss} & \delta, \mathrm{deg} & \delta, \mathrm{mm} & \delta, \mathrm{ss} & \sigma_{\delta}, \mathrm{ss} \\ 569 & 00 & 39 & 44.410 & 0.0008 & 40 & 37 & 54.77 & 0.003 \\ 642 & 00 & 40 & 24.188 & 0.0006 & 41 & 09 & 54.77 & 0.003 \\ 610 & 00 & 41 & 05.703 & 0.0004 & 40 & 59 & 49.02 & 0.002 \\ 198 & 00 & 41 & 21.630 & 0.0003 & 40 & 46 & 31.35 & 0.001 \\ 174 & 00 & 41 & 29.870 & 0.0002 & 40 & 44 & 06.81 & 0.001 \\ 404 & 00 & 41 & 35.899 & 0.0002 & 40 & 59 & 06.55 & 0.002 \\ 277 & 00 & 41 & 37.961 & 0.0002 & 40 & 51 & 40.72 & 0.001 \\ 142 & 00 & 41 & 41.996 & 0.0002 & 40 & 41 & 52.64 & 0.001 \\ 172 & 00 & 41 & 46.260 & 0.0001 & 40 & 44 & 06.31 & 0.003 \\ 353 & 00 & 41 & 50.814 & 0.0001 & 40 & 56 & 56.32 & 0.002 \\ 386 & 00 & 41 & 54.721 & 0.0001 & 40 & 58 & 08.85 & 0.002 \\ 441 & 00 & 41 & 57.823 & 0.0001 & 41 & 02 & 07.84 & 0.002 \\ 387 & 00 & 42 & 01.378 & 0.0001 & 40 & 58 & 45.00 & 0.002 \\ 402 & 00 & 42 & 03.335 & 0.0001 & 40 & 59 & 05.72 & 0.002 \\ 313 & 00 & 42 & 06.497 & 0.0001 & 40 & 54 & 15.14 & 0.001 \\ 254 & 00 & 42 & 09.259 & 0.0001 & 40 & 49 & 35.72 & 0.001 \\ 329 & 00 & 42 & 11.973 & 0.0001 & 40 & 55 & 06.79 & 0.001 \\ 125 & 00 & 42 & 15.150 & 0.0001 & 40 & 40 & 19.10 & 0.001 \\ 255 & 00 & 42 & 19.030 & 0.0001 & 40 & 50 & 11.59 & 0.001 \\ 111 & 00 & 42 & 21.778 & 0.0001 & 40 & 39 & 12.13 & 0.001 \\ 100 & 00 & 42 & 24.896 & 0.0001 & 40 & 38 & 36.09 & 0.002 \\ 217 & 00 & 42 & 27.628 & 0.0001 & 40 & 47 & 00.29 & 0.001 \\ 410 & 00 & 42 & 30.773 & 0.0001 & 41 & 00 & 12.43 & 0.002 \\ 444 & 00 & 42 & 33.520 & 0.0001 & 41 & 01 & 35.54 & 0.002 \\ 23 & 00 & 42 & 35.576 & 0.0001 & 41 & 34 & 29.62 & 0.001 \\ 232 & 00 & 42 & 39.349 & 0.0002 & 40 & 47 & 51.53 & 0.001 \\ 412 & 00 & 42 & 41.750 & 0.0002 & 41 & 00 & 01.70 & 0.002 \\ 433 & 00 & 42 & 45.282 & 0.0002 & 41 & 00 & 43.17 & 0.002 \\ 129 & 00 & 42 & 49.071 & 0.0002 & 40 & 41 & 19.75 & 0.002 \\ 287 & 00 & 42 & 50.318 & 0.0002 & 40 & 52 & 09.56 & 0.001\end{array}$

\section{References}

Deutsch, A.N., Lavdovsky, V.V., Fatchikhin, N.V., Gamalei, N.V., 1953, Izvestiay GAO N150, (in Russian).

Deutsch, A.N., Yakhontov, K.N., 1950, Working catalogue of 32 extragalactic nebulae, GAISH, $M$. 